\title{
DIFFUSION GÉNÉRALE
}

\author{
OCDE/GD(95)66
}

\section{SERIE OCDE}

LES PRINCIPES DE BONNES PRATIQUES DE LABORATOIRE ET LA VERIFICATION DU RESPECT DE CES PRINCIPES

Numéro 2 (révisé)

ORIENTATIONS A L'INTENTION DES AUTORITES DE VERIFICATION EN MATIERE DE BPL

GUIDES REVISES POUR LES SYSTEMES DE VERIFICATION DU RESPECT DES BONNES PRATIQUES DE LABORATOIRE

MONOGRAPHIE SUR L'ENVIRONNEMENT NO. 110

ORGANISATION DE COOPÉRATION ET DE DÉVELOPPEMENT ÉCONOMIQUES Paris 1995

DOCUMENT DISPONIBLE SUR OLIS EN TOTALITÉ, DANS SON FORMAT D'ORIGINE 
SERIE OCDE

LES PRINCIPES DE BONNES PRATIQUES DE LABORATOIRE ET LA VERIFICATION DU RESPECT DE CES PRINCIPES

Numéro 2 (Révisé)

Orientations à l'intention des autorités de vérification en matière de BPL

\section{GUIDES REVISES POUR LES SYSTEMES \\ DE VERIFICATION DU RESPECT DES \\ BONNES PRATIQUES DE LABORATOIRE}

MONOGRAPHIE SUR L'ENVIRONNEMENT NO. 110

Direction de l'Environnement

ORGANISATION DE CO-OPERATION ET DE DEVELOPPEMENT ECONOMIQUES

Paris 1995 
Copyright OCDE, 1995

Les demandes de reproduction ou de traduction doivent être adressées à : M. Le Chef du Service des Publications, OCDE, 2 rue André-Pascal, 75775 Paris Cédex 16, France. 


\section{AVANT-PROPOS}

Aux termes de la Décision du Conseil relative à l'acceptation mutuelle de données [C(81)30(Final)], dont les Principes OCDE relatifs aux Bonnes pratiques de laboratoire ${ }^{1}$ font partie intégrante, l'OCDE a été chargée d'entreprendre des activités visant "à faciliter la mise en oeuvre de méthodes harmonisées au plan international qui puissent assurer le respect des Principes de l'OCDE relatifs aux bonnes pratiques de laboratoire". Par conséquent, afin de promouvoir la mise en oeuvre de procédures comparables de vérification de la conformité et leur acceptation internationale parmi les pays Membres, le Conseil a adopté en 1983 une Recommandation relative à la Reconnaissance mutuelle de la conformité aux Bonnes pratiques de laboratoire [C(83)95(Final)], qui présentait les caractéristiques fondamentales des procédures à suivre lors de la vérification de la conformité.

Un Groupe de travail sur la reconnaissance mutuelle de la conformité aux bonnes pratiques de laboratoire fut établi en 1985, sous la présidence du Professeur V. Silano (Italie), afin de faciliter la mise en oeuvre pratique des actes du Conseil relatifs aux bonnes pratiques de laboratoire, d'élaborer des approches communes pour résoudre les difficultés techniques et administratives liées à la conformité aux BPL ainsi qu'à ses procédures de vérification. Les pays et organisations suivants ont participé au Groupe de travail: l'Australie, la Belgique, le Canada, le Danemark, la République fédérale allemande, la Finlande, la France, l'Italie, le Japon, la Norvège, les Pays-Bas, le Portugal, l'Espagne, la Suède, la Suisse, le Royaume Uni, les Etats-Unis, la Commission des communautés européennes, l'Organisation internationale de la standardisation, la Convention des inspections pharmaceutiques et l'Organisation mondiale de la santé.

Le Groupe de travail a mis au point, entre autres, des Guides pour les systèmes de vérification du respect des bonnes pratiques de laboratoire, qui prennent en considération les besoins des administrations, du personnel et des programmes de vérification du respect des BPL. Ceux-ci ont été publiés pour la première fois en 1988 dans le Rapport final du Groupe de travail ${ }^{2}$. Une version légèrement abrégée a été annexée à la Décision-Recommandation du Conseil sur la Conformité aux principes relatifs aux bonnes pratiques de laboratoire [C(89)87(Final)], qui annule et remplace l'Acte du Conseil de 1983.

Aux termes de la partie III.1 de cette Décision-Recommandation adoptée par le Conseil, le Comité de l'environnement et le Comité de gestion du Programme spécial sur le contrôle des produits chimiques sont chargés de s'assurer que les "Guides pour les systèmes de vérification du respect des bonnes pratiques de laboratoire" et les "Directives pour la conduite d'inspections de laboratoire et de vérifications d'études" figurant aux Annexes I et II, sont mis à jour et complétés, le cas échéant, à la lumière de l'évolution des connaissances et de l'expérience des pays Membres, ainsi que des travaux pertinents menés dans d'autres organisations internationales.

Voir Les Principes de l'OCDE de bonnes pratiques de laboratoire, numéro 1 de cette série OCDE sur les Principes de BPL et la vérification du respect de ces Principes.

2 Rapport final du groupe de travail sur la reconnaissance mutuelle de la mise en conformité aux bonnes pratiques de laboratoire, Monographie de l'OCDE sur l'environnement no. 15, mars 1988. 
La Commission de l'OCDE sur les bonnes pratiques de laboratoire a mis au point des propositions d'amendements de ces Annexes, ainsi que de l'Annexe III qui fournit des "Orientations pour l'échange d'informations concernant les programmes nationaux de vérification du respect des principes de BPL", amendée essentiellement en vue d'inclure un appendice sur des "Orientations à l'intention des autorités de vérification en matière de bonnes pratiques de laboratoire pour la préparation des rapports annuels sur les installations d'essais inspectées". Ces Annexes révisées ont été approuvées par le Conseil le 9 mars 1995, dans une Décision "Modifiant les annexes à la DécisionRecommandation du Conseil sur le respect des principes de bonnes pratiques de laboratoire" [C(95)8(Final)].

La première partie de ce document comporte les Guides révisés pour les systèmes de vérification du respect des BPL tels qu'ils sont apparus dans l'Acte du Conseil de 1989 [C(89)87(Final)] et révisés par le Conseil en 1995 [C(95)8(Final)] ; la Décision-Recommandation, ainsi que l'Annexe III révisée, sont reproduites dans la deuxième partie.

Le présent document annule et remplace la monographie sur l'environnement no. 46 intitulée "Guides pour les systèmes de vérification du respect des bonnes pratiques de laboratoire", publiée en 1992. 


\section{TABLE DES MATIERES}

Première partie Guides révisés pour les systèmes de vérification du 9 respect des bonnes pratiques de laboratoire

Deuxième partie Décision-Recommandation du Conseil sur le respect des Principes de bonnes pratiques de laboratoire [C(89)87(Final)]

Annexe III : Orientations révisées pour l'échange d'informations 23 concernant les programmes nationaux de vérification du respect des Principes de bonnes pratiques de laboratoire 


\title{
PARTIE I :
}

\section{GUIDES REVISES POUR LES SYSTEMES DE VERIFICATION DU RESPECT DES BONNES PRATIQUES DE LABORATOIRE ${ }^{3}$}

\author{
(tels que révisés par le Conseil, le 9 mars 1995)
}

\begin{abstract}
Afin de faciliter l'acceptation mutuelle des données d'essai obtenues en vue de leur soumission aux Autorités responsables des pays Membres de l'OCDE, il est essentiel d'harmoniser dans une certaine mesure les systèmes adoptés afin de vérifier la conformité aux bonnes pratiques de laboratoire, leur équivalence, leur qualité et leur rigueur. Ce document a pour objet de fournir aux pays Membres de l'OCDE des conseils pratiques et détaillés sur la structure, les dispositifs et les systèmes qu'ils doivent adopter pour élaborer les Programmes nationaux de vérification du respect des bonnes pratiques de laboratoire, de manière à ce que leurs Programmes soient acceptables à l'échelon international.
\end{abstract}

Il est entendu que les pays Membres adopteront des Principes de BPL et établiront des systèmes de vérification, conformément aux pratiques légales et administratives en vigueur dans leur pays et aux priorités fixées pour certaines questions telles que les catégories de produits chimiques et les types d'essais entrant dans le domaine d'application, initial et ultérieur, de ces mesures. Etant donné que les pays Membres peuvent établir plus d'une Autorité chargée de contrôler la conformité aux bonnes pratiques de laboratoire en raison du cadre juridique qu'ils ont instauré en vue du contrôle des produits chimiques, il se peut que plus d'un Programme de respect des bonnes pratiques de laboratoire soit établi. Les conseils formulés dans les paragraphes suivants valent, le cas échéant, pour chacune de ces Autorités et chaque Programme de mise en conformité.

\section{DEFINITIONS}

Les définitions des termes données dans les "Principes de l'OCDE de Bonnes Pratiques de Laboratoire" [Annexe 2 à la Décision-Recommandation du Conseil C(81)30(Final)] sont applicables à ce document. S'y ajoutent les définitions suivantes :

Principes de BPL : Principes de bonnes pratiques de laboratoire compatibles avec les Principes de l'OCDE de bonnes pratiques de laboratoire, tels que exposés dans l'Annexe 2 de la Décision du Conseil C(81)30(Final). ${ }^{4}$

Vérification du respect des BPL : inspection périodique d'installations d'essais et/ou vérification d'études réalisées afin de s'assurer du respect des Principes de BPL.

3 Les Guides révisés pour les systèmes de vérification du respect des bonnes pratiques de laboratoire figurent dans la révision de l'Annexe I à la Décision-Recommandation sur le respect des Principes de bonnes pratiques de laboratoire [C(89)87(Final)]. Pour le texte de cet Acte du Conseil, voir page 17 de cette publication.

$4 \quad$ Voir Les Principes de l'OCDE de bonnes pratiques de laboratoire, numéro 1 de cette série OCDE sur les Principes de BPL et la vérification du respect de ces Principes. 
Programme (national) de respect des BPL : dispositif particulier établi par un pays Membre pour vérifier le respect des BPL par les installations d'essais situées sur son territoire, au moyen d'inspections et de vérifications d'études.

Autorité (nationale) de vérification en matière de BPL : organisme créé dans un pays Membre pour contrôler le respect des BPL par les installations d'essais situées sur son territoire et remplir d'autres fonctions du même ordre relatives aux BPL, qui pourront être définies au niveau national. Il est entendu que plusieurs organismes de ce type peuvent être créés dans un pays Membre.

Inspection d'installation d'essais : examen sur place des procédures et des méthodes appliquées dans l'installation d'essais afin d'évaluer le degré de conformité aux Principes de BPL. Au cours des inspections, la structure administrative et les modes opératoires normalisés de l'installation d'essais sont examinés, le personnel technique d'encadrement est interviewé, la qualité ainsi que l'intégrité des données obtenues par l'installation sont évaluées, et il en est rendu compte dans un rapport.

Vérification d'étude : comparaison des données brutes et des rapports qui y sont associés avec le rapport provisoire ou final, en vue de déterminer si les données brutes ont été notifiées avec exactitude, de vérifier si les essais ont été menés conformément au plan d'étude et aux modes opératoires normalisés, d'obtenir des informations complémentaires ne figurant pas dans le rapport, et d'établir si les méthodes utilisées pour obtenir les données ne risquaient pas d'entacher leur validité.

Inspecteur : personne qui réalise l'inspection de l'installation d'essais et la vérification d'étude pour le compte de l'Autorité (nationale) de vérification en matière de BPL.

Degré de conformité aux BPL : degré d'adhésion aux principes de BPL d'une installation d'essais, qui est évalué par l'Autorité (nationale) de vérification en matière de BPL.

Autorité réglementaire : organisme national ayant juridiquement compétence pour les questions touchant au contrôle des produits chimiques.

\section{COMPOSANTES DES SYSTEMES DE VERIFICATION DU RESPECT DES BPL}

\section{Administration}

Un Programme (national) de respect des BPL doit relever des compétences d'un organisme constitué dans les règles, ayant une identité juridique, pourvu d'un personnel adéquat et fonctionnant dans un cadre administratif bien précis.

Les pays Membres doivent :

- s'assurer que l'Autorité (nationale) de vérification en matière de BPL est directement responsable des activités menées par une "équipe" appropriée d'inspecteurs, ayant les compétences techniques et scientifiques nécessaires, ou est responsable en dernier ressort des activités de ladite "équipe" ;

- publier les documents concernant l'adoption des Principes de BPL sur leur territoire ;

- publier des documents fournissant des informations détaillées sur le Programme (national) de respect des BPL, y compris des informations sur le cadre juridique ou administratif 
de ce programme et des références aux lois promulguées, textes normatifs (règlements, codes d'usage, par exemple), manuels d'inspection, notes d'orientation, périodicité des inspections et/ou critères retenus pour en établir le calendrier etc. ;

- conserver des archives sur les inspections d'installations d'essais (et leur degré de conformité aux BPL), et les vérifications d'études, à des fins tant nationales qu'internationales.

\section{Confidentialité}

Les Autorités (nationales) de vérification en matière de BPL ont accès à des informations ayant une valeur commerciale et, éventuellement, peuvent même avoir besoin de retirer d'une installation d'essais des documents sensibles sur le plan commercial, ou s'y référer de façon détaillée dans leurs rapports.

Les pays Membres doivent :

- prendre des dispositions appropriées pour empêcher toute divulgation, non seulement par les inspecteurs mais aussi par toute autre personne ayant accès à des informations confidentielles du fait d'activités de vérification de la conformité aux BPL ;

- s'assurer que, excepté le cas où toutes les informations sensibles sur le plan commercial et confidentiel ont été retirées, les rapports d'inspections des installations d'essais et de vérifications d'études ne sont mis à la disposition que des Autorités réglementaires et, le cas échéant, des installations d'essais inspectées ou concernées par les vérifications d'études et/ou des commettants des études.

\section{Personnel et formation}

Les Autorités (nationales) de vérification en matière de BPL doivent :

— s'assurer qu'il y a un nombre suffisant d'inspecteurs

Le nombre d'inspecteurs requis dépend :

i) du nombre d'installations d'essais incluses dans le Programme (national) de respect des BPL ;

ii) de la périodicité à laquelle le degré de conformité des installations d'essais doit être évalué ;

iii) du nombre et de la complexité des études entreprises par ces installations d'essais ;

iv) du nombre d'inspections ou de vérifications particulières, expressément demandées par les Autorités réglementaires.

- s'assurer que les inspecteurs possèdent des qualifications suffisantes et une formation adéquate 
Les inspecteurs doivent avoir des qualifications et une expérience pratique dans l'ensemble des disciplines intéressant les essais de produits chimiques. Les Autorités (nationales) de vérification en matière de BPL doivent :

i) faire en sorte que des dispositions soient prises pour donner une formation appropriée aux inspecteurs de BPL, compte tenu des qualifications et de l'expérience de chacun ;

ii) encourager les consultations, y compris les activités conjointes de formation, le cas échéant, avec le personnel des Autorités (nationales) de vérification en matière de BPL des autres pays Membres en vue de favoriser l'harmonisation, au niveau international, de l'interprétation et de l'application des Principes relatifs aux BPL et de la vérification du respect de ces Principes.

- s'assurer que le personnel d'inspection, y compris les experts employés sous contrat, n'a pas un intérêt financier ou autre dans les installations d'essais inspectées, les études vérifiées ou dans les entreprises participant au financement de ces études

- fournir aux inspecteurs un moyen approprié d'identification (par exemple, une carte d'identité).

Les inspecteurs peuvent :

- faire partie du personnel permanent de l'Autorité (nationale) de vérification en matière de BPL;

- faire partie du personnel permanent d'un organisme distinct de l'Autorité (nationale) de vérification en matière de BPL; ou

- être employés sous contrat, ou sous une autre forme, par l'Autorité (nationale) de vérification en matière de BPL pour réaliser des inspections d'installations d'essais ou des vérifications d'études.

Dans ces deux derniers cas, l'Autorité (nationale) de vérification en matière de BPL doit avoir en dernier ressort la responsabilité de déterminer le degré de conformité aux BPL des installations d'essais et la qualité et l'acceptabilité d'une vérification d'étude, et de prendre toute mesure pouvant s'avérer nécessaire, compte tenu des résultats des inspections d'installations d'essais ou des vérifications d'études.

\section{Programmes (nationaux) de respect des BPL}

La vérification du respect des BPL vise à établir si les installations d'essais ont appliqué, pour la conduite de leurs études, les Principes de bonnes pratiques de laboratoire et s'ils sont en mesure de garantir une qualité suffisante pour les données obtenues. Comme cela est indiqué ci-dessus, les pays Membres doivent publier des informations détaillées sur leurs Programmes (nationaux) de mise en conformité aux BPL. Ces informations doivent entre autres : 
Un Programme (national) de respect des BPL peut porter seulement sur une gamme restreinte de produits chimiques, par exemple les produits chimiques industriels, les pesticides, les produits pharmaceutiques, etc., ou peut inclure tous les produits chimiques. La portée du contrôle doit être précisée en ce qui concerne tant les catégories de produits chimiques que les types d'essais en faisant l'objet, qui peuvent être notamment physiques, chimiques, toxicologiques et/ou écotoxicologiques.

- donner une indication sur le mécanisme par lequel les installations d'essais sont inscrites au programme de respect des $B P L$

L'application des Principes de BPL aux données concernant l'innocuité des produits pour la santé et l'environnement, qui ont été obtenues à des fins réglementaires, peut être obligatoire. Il convient de mettre en place une procédure en vertu de laquelle les installations d'essais pourront faire vérifier par l'Autorité (nationale) de vérification en matière de BPL compétente, leur conformité aux BPL.

- contenir des informations sur les catégories d'inspections d'installations d'essais et de vérifications d'études

Un Programme (national) de respect des BPL doit comprendre :

i) des dispositions appropriées pour des inspections d'installations d'essais. Ces inspections comprennent à la fois une inspection générale de l'installation d'essais et la vérification d'une ou de plusieurs étude(s) en cours ou déjà achevée(s) ;

ii) des dispositions appropriées pour des inspections d'installations d'essais et vérifications d'études spéciales faites à la demande d'une Autorité réglementaire - par exemple les inspections et vérifications déclenchées par une demande formulée à la suite de la soumission des données à une Autorité réglementaire.

- définir les pouvoirs des inspecteurs quant à leur accès aux installations d'essais et aux données détenues par ceux-ci (y compris aux spécimens, aux modes opératoires normalisés, à toute autre documentation, etc.)

Si les inspecteurs ne veulent pas le plus souvent pénétrer dans les installations d'essais contre le gré de la direction de l'installation, il peut se produire que l'accès à l'installation d'essais et aux données soit essentiel pour protéger la santé publique ou l'environnement. Les pouvoirs, dont dispose l'Autorité (nationale) de vérification en matière de BPL, doivent être définis pour de telles circonstances.

- décrire les procédures d'inspection d'installation d'essais et de vérification d'études pour contrôler la conformité aux BPL

La documentation doit indiquer les méthodes qui seront employées pour examiner à la fois la structure administrative et les conditions dans lesquelles les études sont préparées, réalisées, contrôlées et enregistrées. Les Directives applicables à ces procédures figurent dans les Directives pour la conduite d'inspections d'installations d'essais et de 
vérifications d'études (numéro 3 de cette série OCDE sur les Principes de BPL et la vérification du respect de ces Principes).

- décrire les mesures susceptibles d'être prises dans le cadre du suivi des inspections d'installations d'essais et vérifications d'études

\section{Suivi des inspections d'installations d'essais et des vérifications d'études}

Lorsqu'une inspection d'installation d'essais ou vérification d'étude a été achevée, l'inspecteur doit établir un rapport écrit sur ses conclusions.

Les pays Membres doivent prendre des mesures lorsque des écarts par rapport aux Principes de BPL sont constatés pendant ou après une inspection d'installation d'essais ou une vérification d'étude. Ces mesures doivent être décrites dans des documents émanant de l'Autorité (nationale) de vérification en matière de BPL.

Si une inspection d'installation d'essais ou une vérification d'étude ne révèle que des écarts mineurs par rapport aux Principes de BPL, l'installation est tenue de rectifier de tels écarts mineurs. L'inspecteur peut avoir besoin, à un moment opportun, de retourner sur les lieux pour vérifier que des rectifications ont été apportées.

Si aucun écart n'est constaté, ou seulement un écart mineur, les Autorités (nationales) de vérification en matière de BPL peuvent :

- publier une déclaration indiquant que l'installation d'essais a été inspectée et que son fonctionnement a été estimé conforme aux Principes de BPL. La date de l'inspection doit y figurer, et, le cas échéant, les catégories d'essais inspectés dans l'installation d'essais à ce moment là devront être inclus ; ces déclarations peuvent être utilisées pour fournir des informations aux Autorités (nationales) de vérification en matière de BPL dans d'autres pays Membres ;

et/ou

- communiquer à l'Autorité réglementaire qui a demandé la vérification d'étude, un rapport détaillé sur les conclusions.

Quand de graves écarts sont constatés, les mesures que peuvent prendre les Autorités (nationales) de vérification en matière de BPL dépendent des circonstances particulières à chaque cas et des dispositions légales ou administratives en vertu desquelles le contrôle de la mise en conformité aux BPL a été institué dans chaque pays. Sans qu'elles ne se limitent à cela, les mesures susceptibles d'être prises consistent notamment à :

- publier une déclaration fournissant des détails sur les défaillances ou anomalies constatées et susceptibles d'altérer la validité des études conduites dans l'installation d'essais ;

— publier une recommandation à l'intention de l'Autorité réglementaire de rejeter une étude ; 
- suspendre les inspections ou vérifications d'études d'une installation d'essais et, par exemple, lorsque cela est possible sur le plan administratif, exclure l'installation d'essais du Programme (national) de respect des BPL ou de toute liste ou de tout registre existants d'installations d'essais soumises aux inspections en matière de BPL ;

- exiger qu'une déclaration indiquant de façon détaillée les écarts soit jointe aux rapports d'études spécifiques ;

- introduire une action devant les tribunaux, dès lors que la situation le justifie et que les procédures légales ou administratives le permettent.

\section{Procédures d'appel}

Les problèmes ou divergences de vues surgissant entre les inspecteurs et la direction des installations d'essais sont normalement résolus pendant l'inspection de l'installation d'essais ou la vérification d'étude. Toutefois, il n'est pas toujours possible de parvenir à un accord. Il faut faire en sorte qu'il existe une procédure donnant la possibilité à l'installation d'essais d'exposer son point de vue concernant les conclusions d'une inspection d'installation d'essais ou d'une vérification d'étude en vue de contrôler la conformité aux BPL, et/ou les mesures que l'Autorité de vérification en matière de BPL se propose de prendre. 


\section{DECISION-RECOMMANDATION DU CONSEIL sur le respect des principes de bonnes pratiques de laboratoire [C(89)87(Final)]}

(adoptée par le Conseil lors de sa 717ème session le 2 octobre 1989)

\section{Le Conseil,}

Vu les articles 5 a) et 5 b) de la Convention relative à l'Organisation de Coopération et de Développement Economiques, en date du 14 décembre 1960 ;

Vu la Recommandation du Conseil, en date du 7 juillet 1977, fixant les lignes directrices pour la procédure et les éléments nécessaires à l'évaluation des effets potentiels des produits chimiques sur l'homme et dans l'environnement [C(77)97(Final)] ;

Vu la Décision du Conseil, en date du 12 mai 1981, relative à l'acceptation mutuelle des données pour l'évaluation des produits chimiques $[\mathrm{C}(81) 30$ (Final)] et, en particulier, la Recommandation invitant les pays Membres, lors d'essais de produits chimiques, à appliquer les Principes de l'OCDE de bonnes pratiques de laboratoire, présentés dans l'Annexe 2 à cette Décision ;

Vu la Recommandation du Conseil du 26 juillet 1983 relative à la reconnaissance mutuelle de la mise en conformité aux bonnes pratiques de laboratoire [C(83)95(Final)] ;

$\mathrm{Vu}$ les conclusions de la troisième Réunion à haut niveau du Groupe des produits chimiques (OCDE, Paris, 1988) ;

Considérant la nécessité d'assurer que les données d'essais sur des produits chimiques fournies aux autorités responsables à des fins d'évaluation et pour d'autres utilisations liées à la protection de la santé humaine et de l'environnement, sont de haute qualité, valides et fiables ;

Considérant la nécessité de réduire autant que possible la répétition des essais de produits chimiques et donc d'utiliser de façon plus efficace le nombre limité de laboratoires et de spécialistes et de diminuer le nombre d'animaux utilisés dans les essais ;

Considérant que la reconnaissance de ces systèmes de vérification du respect des bonnes pratiques de laboratoire facilitera l'acceptation mutuelle des données et de ce fait, limitera la répétition d'essais de produits chimiques ;

Considérant que la reconnaissance de ces systèmes de vérification repose sur la compréhension des systèmes établis dans le pays Membre où les données sont obtenues et sur la confiance à leur accorder ;

Considérant que des approches harmonisées de la vérification faciliteraient considérablement l'établissement de la confiance nécessaire que les systèmes établis dans d'autres pays doivent inspirer ; 
Sur la proposition de la Réunion conjointe du Comité de gestion du Programme spécial sur le contrôle des produits chimiques et du Groupe des produits chimiques, approuvée par le Comité de l'environnement ;

\section{PARTIE I}

\section{Principes de BPL et vérification du respect de ces principes}

1. DECIDE : les pays Membres dans lesquels, à des fins d'évaluation liée à la protection de la santé et de l'environnement, des essais de produits chimiques sont réalisés conformément à des principes de bonnes pratiques de laboratoire compatibles avec les Principes de l'OCDE de bonnes pratiques de laboratoire tels qu'ils sont présentés dans l'Annexe 2 de la Décision du Conseil C(81)30(Final) (ci-après dénommés "Principes de BPL"),

i) instituent au niveau national des systèmes de vérification du respect des Principes de BPL, fondés sur des inspections de laboratoire et sur des vérifications d'études ;

ii) désignent une ou plusieurs autorités pour remplir les fonctions requises par ces systèmes de vérification ; et

iii) exigent de la direction des laboratoires qu'elle produise, le cas échéant, une déclaration établissant qu'un essai a été effectué conformément aux Principes de BPL et à toutes autres dispositions contenues dans les réglementations ou les procédures administratives nationales relatives aux bonnes pratiques de laboratoire.

2. RECOMMANDE que les pays Membres, en instituant en en mettant en oeuvre des systèmes de vérification du respect des Principes de BPL, se conforment aux "Guides pour les systèmes de vérification du respect des bonnes pratiques de laboratoire" et aux "Directives pour la conduite d'inspections de laboratoire et de vérifications d'études", figurant respectivement aux Annexes I et II qui font partie intégrante de la présente Décision-Recommandation. ${ }^{5}$

\section{PARTIE II}

\section{$\underline{\text { Reconnaissance, entre pays Membres, du respect des BPL }}$}

1. DECIDE : Les pays Membres reconnaissent l'assurance donnée par un autre pays Membre que les données d'essais ont été obtenues conformément aux Principes de BPL, si cet autre pays Membre se conforme à la Partie I ci-dessus et à la Partie II, paragraphe 2, ci-dessous.

2. DECIDE : Les pays Membres, à des fins de reconnaissance de l'assurance dont il est question au paragraphe 1 ci-dessus :

i) désignent une ou plusieurs autorité(s) chargée(s) d'assurer la liaison sur le plan international et de remplir d'autres fonctions liées à la reconnaissance, telles que celles

\footnotetext{
La révision de l'Annexe I à l'Acte du Conseil [présentée dans C(95)8(Final)] constitue la première partie (pages 9 à 15) de cette publication. L'on trouvera l'Annexe II dans Directives révisées pour la conduite d'inspections de laboratoires et de vérifications d'études, numéro 3 (révisé) de cette série OCDE sur les Principes de BPL et la vérification du respect de ces Principes (Monographie sur l'environnement No. 111).
} 
mentionnées dans la présente Partie et dans les Annexes à la présente DécisionRecommandation ;

ii) échangent avec d'autres pays Membres des informations utiles relatives à leurs systèmes de vérification, conformément aux orientations figurant à l'Annexe III $^{6}$, qui fait partie intégrante de la présente Décision-Recommandation; et

iii) mettent en place des procédures permettant, si cela est justifié, que des informations concernant le respect des BPL par un laboratoire situé sur leur territoire (y compris des informations concernant un essai particulier) puissent être obtenues par un autre pays Membre.

3. DECIDE : La Recommandation du Conseil relative à la reconnaissance mutuelle de la mise en conformité aux bonnes pratiques de laboratoire [C(83)95(Final)] est abrogée.

\section{PARTIE III}

\section{Activités futures de l'OCDE}

1. CHARGE le Comité de l'environnement et le Comité de gestion du Programme spécial sur le contrôle des produits chimiques de s'assurer que les "Guides pour les systèmes de vérification du respect des bonnes pratiques de laboratoire" et les "Directives pour la conduite d'inspections de laboratoire et de vérifications d'études" figurant aux Annexes I et $\mathrm{II}^{7}$ sont mis à jour et complétés, le cas échéant, à la lumière de l'évolution des connaissances et de l'expérience des pays Membres, ainsi que des travaux pertinents menés dans d'autres organisations internationales.

2. CHARGE le Comité de l'environnement et le Comité de gestion du Programme spécial sur le contrôle des produits chimiques de mener un programme de travail destiné à faciliter la mise en oeuvre de la présente Décision-Recommandation, et de s'assurer que les questions techniques et administratives associées à l'application des Principes de BPL et à la mise en oeuvre de systèmes de vérification du respect de ces bonnes pratiques de laboratoire, font l'objet d'un échange continu d'informations et d'expériences.

3. CHARGE le Comité de l'environnement et le Comité de gestion du Programme spécial sur le contrôle des produits chimiques d'examiner les actions entreprises par les pays Membres en application de la présente Décision-Recommandation.

6 Pour la révision de l'Annexe III à l'Acte du Conseil [Orientations révisées pour l'échange d'informations concernant les programmes nationaux de vérification du respect des Principes de bonnes pratiques de laboratoire, présentées dans C(95)8(Final)], voir page 23 de cette publication.

$7 \quad$ Voir note 5 , page 18 
Annexe I à C(89)87(Final)/Révisée dans $\mathrm{C}(95) 8$ (Final)

GUIDES REVISES POUR LES SYSTEMES DE VERIFICATION DU RESPECT DES BONNES PRATIQUES DE LABORATOIRE

voir pages 9 à 15

***

Annexe II à C(89)87(Final)/Révisée dans C(95)8(Final)

DIRECTIVES REVISEES POUR LA CONDUITE D'INSPECTIONS DE LABORATOIRES ET DE VERIFICATIONS D'ETUDES

voir la série OCDE sur les Principes de BPL

et la vérification du respect de ces Principes, no. 3 (révisé)

(Monographie sur l'environnement No. 111) 


\title{
ORIENTATIONS REVISEES POUR L'ECHANGE D'INFORMATIONS CONCERNANT LES PROGRAMMES NATIONAUX DE VERIFICATION DU RESPECT DES PRINCIPES DE BONNES PRATIQUES DE LABORATOIRE
}

\author{
(telles que révisées par le Conseil, le 9 mars 1995)
}

La Partie II paragraphe 2 de l'Acte du Conseil contient une Décision aux termes de laquelle les pays Membres devront échanger des informations concernant leurs programmes de vérification du respect des Principes de BPL. Cette Annexe fournit des orientations sur les types d'information qu'il convient d'échanger. Tandis que des informations concernant tous les aspects traités dans les "Guides pour les systèmes de vérification du respect des bonnes pratiques de laboratoire" (Annexe I) sont utiles pour comprendre les programmes de vérification d'un autre pays, certains types d'information revêtent une importance particulière. Ceux-ci concernent :

— les Principes de BPL adoptés au plan national ;

- la portée du programme national de vérification du respect des Principes de BPL en termes de catégories de produits chimiques et de types d'essais entrant dans le domaine d'application ;

- l'identité, le statut juridique et la structure administrative de l'Autorité (nationale) ou des Autorités de vérification en matière de BPL ;

- les procédures suivies au cours des inspections d'installations d'essais et des vérifications d'études ainsi que la périodicité des inspections et/ou les critères retenus pour en établir le calendrier ;

— le nombre et la qualification des inspecteurs ;

- les mesures à la disposition de l'Autorité (des Autorités) (nationale[s]) de vérification en matière de BPL en cas de non conformité, y compris la possibilité de communiquer aux autres pays Membres, lorsqu'il y a lieu, les résultats des inspections d'installations d'essais et des vérifications ;

— les dispositions prises pour protéger la confidentialité des informations ;

- les procédures applicables au lancement, au déroulement et à l'établissement des rapports d'inspections d'installations d'essais et de vérifications d'études, à la demande d'autres pays Membres ;

- les procédures suivies pour obtenir des informations sur les installations d'essais qui ont fait l'objet d'une inspection par une Autorité (nationale) de vérification en matière de BPL d'un autre pays Membre, notamment sur le degré de conformité de ces installations ; et 
- la nature des déclarations des installations d'essais établissant que les études ont été réalisées conformément aux Principes de BPL.

Si de graves écarts sont relevés qui peuvent avoir affecté certaines études, l'Autorité (nationale) de vérification en matière de BPL doit envisager la nécessité de transmettre ses conclusions aux Autorités réglementaires compétentes ou aux Autorités (nationales) de vérification en matière de BPL d'autres pays Membres.

Les noms des installations d'essais soumises à des inspections dans le cadre d'un Programme (national) de respect des BPL, leur degré de conformité aux Principes relatifs aux BPL et les dates des inspections, doivent être mis à la disposition des Autorités (nationales) de vérification en matière de BPL d'autres pays Membres sur demande (voir les "Orientations à l'intention des autorités de vérification en matière de bonnes pratiques de laboratoire pour la préparation des rapports annuels sur les installations d'essais inspectées", présentées dans l'Appendice à cette même Annexe).

Il est possible que les autres pays ne reconnaissent pas immédiatement les programmes nationaux de vérification du respect des Principes de BPL. Les pays Membres doivent être disposés à résoudre les vrais problèmes en faisant preuve d'un esprit de coopération. Il se peut qu'un pays Membre ne soit pas en mesure d'apprécier l'acceptabilité des programmes de vérification du respect des BPL d'un autre pays en se fondant uniquement sur l'échange d'informations écrites. Dans ce cas, les pays Membres peuvent obtenir l'assurance recherchée en menant des consultations et en procédant à des échanges de vues avec les Autorités (nationales) de vérification en matière de BPL concernées. A cet égard, l'OCDE offre un forum permettant de débattre et de résoudre des problèmes liés à l'harmonisation et à l'acceptation internationales des programmes de vérification du respect des BPL.

Pour faciliter les contacts au niveau international et l'échange permanent d'informations, la création d'une seule Autorité de vérification en matière de BPL, englobant toutes les activités se rapportant aux bonnes pratiques de laboratoire dans un pays Membre, présente des avantages évidents. Quand il existe plusieurs Autorités, les pays Membres doivent s'assurer qu'elles fonctionnent de manière concordante, et ont des programmes de respect des BPL analogues. Les pays Membres doivent déterminer l'Autorité ou les Autorités chargées des contacts au niveau international.

Il peut se produire qu'une autorité réglementaire nationale d'un pays Membre ait besoin de demander des informations sur le degré de conformité aux BPL d'une installation d'essais située dans un autre pays Membre. En de rares occasions et lorsqu'il existe des raisons valables, une vérification d'étude particulière peut être demandée par l'Autorité réglementaire d'un autre pays Membre. Des dispositions doivent être prévues pour que ces demandes soient satisfaites et pour que les résultats soient notifiés à l'Autorité réglementaire qui en a fait la demande.

Il convient d'établir des contacts formels au niveau international pour l'échange d'informations entre les Autorités de vérification en matière de BPL. Il ne faut pas en conclure toutefois que cela empêche tout contact informel entre les Autorités réglementaires et l'Autorité de vérification en matière de BPL existant dans un autre pays Membre, dans la mesure où les pays Membres concernés acceptent de tels contacts.

Les Autorités nationales doivent tenir compte du fait que les autorités d'autres pays Membres pourraient vouloir assister à une inspection d'installation d'essais ou une vérification d'étude qu'elles ont expressément demandée, ou peuvent souhaiter que de(s) représentant(s) du pays Membre souhaitant une inspection d'installation d'essais ou une vérification particulière soit (soient) présent(s) à cette inspection ou vérification d'étude. Dans ce cas, les pays Membres doivent permettre aux inspecteurs 
d'un autre pays Membre de participer à des inspections d'installations et des vérifications d'études réalisées par les Autorités de vérification en matière de BPL de leur pays. 


\section{Appendice à l'Annexe III à C(89)87(Final)/Révisée dans C(95)8(Final)}

\section{ORIENTATIONS A L'INTENTION DES AUTORITES DE VERIFICATION EN MATIERE DE BONNES PRATIQUES DE LABORATOIRE POUR LA PREPARATION DES RAPPORTS ANNUELS SUR LES INSTALLATIONS D'ESSAIS INSPECTEES}

Des rapports annuels sur les inspections en matière de BPL doivent être diffusés auprès des Membres de la Commission, ainsi que du Secrétariat, chaque année, avant la fin du mois de mars. L'ensemble des renseignements suivants doit permettre l'harmonisation des rapports annuels échangés entre les autorités nationales de vérification des BPL.

1. Identification de l'installation inspectée : Des informations suffisantes doivent être données afin d'identifier l'installation sans équivoque - le nom de l'installation, de la ville et du pays où elle est située, y compris les inspections conduites à l'étranger.

2. Dates d'inspections et de décisions : Le mois et la date de l'inspection et, s'il y a lieu, la date de la décision finale quant à l'état de conformité aux BPL.

3. Nature de l'inspection : Il doit être clairement indiqué s'il s'agit d'une inspection en matière de BPL complète ou d'une simple vérification d'étude, s'il s'agit d'une inspection routinière ou non, et quelles sont les autres autorités qui y ont participé.

4. Domaines de compétence de l'installation inspectée : Etant donné que le respect des BPL est lié aux essais effectués dans une installation, le(s) domaine(s) de compétence des installations d'essais inspectées doit(doivent) être indiqué(s) dans les rapports annuels, à l'aide des catégories générales suivantes :

1) essais physico-chimiques

2) études de toxicité

3) études de mutagénicité

4) études écotoxicologiques sur les organismes marins et terrestres

5) études portant sur le comportement dans l'eau, dans le sol et dans l'air ; bioaccumulation

6) études portant sur les résidus

7) études portant sur les effets sur les mésocosmes et les écosystèmes naturels

8) essais portant sur les examens analytiques et cliniques

9) autres études, spécifier.

Il faut souligner que ces catégories sont à utiliser de manière souple, au cas par cas, l'objectif étant ici de fournir des informations relatives au respect des BPL par les installations d'essais, qui soient utiles à d'autres autorités nationales de vérification en matière de BPL.

5. Statut de conformité aux BPL : Les trois catégories d'observations suivantes doivent être utilisées pour rendre compte du statut de conformité aux BPL par les installations :

- respect

- non respect

— "en instance" (avec explications à l'appui) 
Etant donné que l'expression "en instance" peut être interprétée différemment par les Pays membres, dont les systèmes administratifs et juridiques varient et ne prévoient donc pas l'usage harmonisé de ce terme, le statut de conformité aux BPL dit "en instance" doit être accompagné d'explications dans les rapports annuels nationaux sur les installations d'essais inspectées. Ces explications pourraient être formulées de la façon suivante : "réinspection en instance", "réponses de l'installation d'essais en instance", "accomplissement des procédures administratives en instance", etc.

6. Commentaires : De plus amples commentaires peuvent être apportés, s'il y a lieu.

7. Carences majeures: A tout le moins, les études individuelles pour lesquelles une vérification d'étude a révélé de sérieux manquements au respect des bonnes pratiques de laboratoire, et qui ont par conséquent été rejetées par les autorités destinataires, doivent être consignées dans les rapports annuels sur les installations d'essais inspectées. Toutefois, étant donné que beaucoup d'études sont soumises aux autorités de plusieurs pays en même temps, il est recommandé que ce type d'informations soit diffusé auprès des autorités nationales, aussi rapidement que possible, sur une base ad hoc, en plus des rapports annuels, lorsqu'il y a lieu.

8. Déclarations de conformité aux BPL : Lorsque les autorités nationales de vérification fournissent aux installations les déclarations de conformité aux BPL, ces déclarations doivent utiliser la même terminologie et les mêmes catégories d'observations que les rapports annuels.

9. Diffusion des rapports annuels : Les rapports sur les installations d'essais inspectées doivent être diffusés annuellement, avant la fin du mois de mars, auprès des Membres de la Commission des bonnes pratiques de laboratoire et du Secrétariat de l'OCDE. Ces informations peuvent être rendues publiques à la demande. 